\section{0-01. ANDRO-IVF: a novel protocol for poor responders to controlled ovarian stimulation for IVF}

\author{
L Bercaire ${ }^{1}$, SMB Nogueira1, PCM Lima1, ${ }^{1}$ R Alves ${ }^{1}$, N \\ Donadio', A Dzik¹, M Cavagna1, R Fanchin² \\ ${ }^{1}$ CRSM - Hospital Pérola Byington, São Paulo - SP, Brazil. \\ ${ }^{2}$ IVF Centre, Hospital Foch, Suresnes, France.
}

Objective: Evaluate a novel protocol for poor responders based on androgenization, in order to improve ovarian response. The endpoints were: number of oocytes and mature oocytes collected, fertilization and pregnancy rates.

Methods: Prospective crossover study including poor responders in previous ovarian stimulation submmited to a novel protocol named as ANDRO-IVF. It includes androgenization with transdermal testosterone (AndroGel ${ }^{\circledR}$ ) $25 \mathrm{mg}$, oral letrozole $2.5 \mathrm{mg}$ and subcutaneous hCG 2,500IU; cycle control was performed with estradiol valerate and micronized progesterone and ovarian stimulation with FSH $300 \mathrm{UI}$ and LH 150UI, GnRH antagonist and hCG 5,000UI.

Results: Fourteen poor-responder patients were enrolled. One patient did not meet inclusion criteria. Thirteen patients had been summited to previous cycles with standard protocol and the same patients received therefore ANDRO-IVF Protocol.

Standard Protocol: Mean age was 35.30. Cancellation rate was $57.14 \%$ and a mean of 2 oocytes/patient was retrieved. Fertilization rate was $41.66 \%$. Three patients performed embryo transfer and no pregnancy was acomplished.

ANDRO-IVF Protocol: Mean age was 35.83. Cancellation rate was $7.14 \%$. Mean oocytes retrieved was 5.58 and mature oocytes was 3.91 /patient. ICSI was performed at $84.61 \%$ of patients and we transfered a number of 1.5 embryo/patient. Fertility rate was $54.93 \%$. Cumulative pregnancy rate was $16.66 \%$. Mean duration of stimulation was 9.77 days.

Conclusion: ANDRO-IVF foresees androgenization by increasing serum and intrafollicular androgen concentrations and preventing androgens aromatization. This protocol seems to improve clinical outcomes in poor responder patients, such as number of oocytes and clinical pregnancy. Further RCT studies are needed to confirm these findings.

\section{0-02. The impact of high pro- gesterone levels on the day of HCG administration in Assist- ed Human Reproduction Treat- ments}

\author{
MS Tanada ${ }^{1}$, IH Yoshida ${ }^{1,2}$, M Santos ${ }^{1,2}$, CZ Berton ${ }^{1}$, E \\ Souto $^{1}$, WP de Carvalho ${ }^{1,2}$, E B Cordts ${ }^{1,2}$, CP Barbosa ${ }^{1,2}$ \\ ${ }^{1}$ Instituto Ideia Fértil de Saúde Reprodutiva, Santo André \\ - SP, Brazil. \\ ${ }^{2}$ Faculdade de Medicina do ABC, Santo André - SP, Brazil.
}

Objective: Progesterone is a female steroid hormone, which acts on the entire physical and emotional of the woman and endometrium. Because of the small number of scientific papers available on the effects of progesterone levels on embryo development and quality, our study aims to determine a cutoff value of serum progesterone levels on the day of HCG.

Methods: We studied 145 cycles, of which 885 oocytes and 613 embryos were obtained. Serum levels of progesterone were measured on the day of HCG in all patients. The data were collected through the analysis of medical records. The values were tabulated and the statistical analysis of the qualitative variables was performed using the chi-square test, and the Mann-Whitney test was used to evaluate the quantitative variables.

Results: Statistical analysis did not demonstrate a statistically significant association between progesterone serum levels and laboratorial reproductive variables. However, in relation to oocyte maturity, it was observed that, when progesterone levels were above $1.3 \mathrm{ng} / \mathrm{mL}$, the probability of oocytes being immature increased by $12.7 \%$. Regarding the variable of embryos classified as "top quality" in D3, there was an increase in the rate of embryo fragmentation proportional to the increase of levels of progesterone $(12.23 \%)$.

Conclusion: High progesterone levels appear to be correlated with an increase in the rate of embryo fragmentation, but high serum levels of the hormone on the day of HCG have no influence on laboratory variables, not allowing us to classify it as a negative marker of embryo development.

\section{0-03. Cryopreserved human seminal samples can be stored at $-80^{\circ} \mathrm{C}$ ?}

\author{
CR Vaz ${ }^{1}$, T Lamim ${ }^{1}$, RA Salvador ${ }^{1}$, APB Batschauer ${ }^{1}$, \\ VLL Amaral' ${ }^{1}$, D Til $^{1}$
}

${ }^{1}$ Laboratório de Biotecnologia da Reprodução (LBR), Universidade do Vale do Itajaí (UNIVALI), Itajaí, Santa Catarina, Brazil.

Objective: To evaluate the effect of storage time for samples of human seminal cryopreserved kept in the freezer with a controlled temperature of $-80^{\circ} \mathrm{C}$ on the sperm viability after thawing.

Methods: We used 20 seminal samples. Each sample was cryopreserved in 10 fingers, which were divided into five groups: one group kept in cryogenic canister (control) throughout the experiment, and four groups kept in freezer VIP UItra Low MDF-U76VPE with controlled temperature of $-80^{\circ} \mathrm{C}$, for $24,48,72$ and 96 hours, respectively, after the exposure time samples were stored in cryogenic canister for after being thawed. The analyzed parameters were: motility, vitality and mitochondrial activity.

Results: After thawing were observed decreased values of motility, vitality and mitochondrial activity, when comparing the tested groups with the control group, as well as a progressive reduction of analyzed parameters between the times evaluated.

Conclusion: cryopreserved seminal samples maintenance at $-80^{\circ} \mathrm{C}$ is potentially harmful to sperm viability, suffering major damage with longer exposure times. 


\section{0-04. Can the morphology of trophectoderm act as a predictive marker for euploidy?}

IH Yoshida ${ }^{1,2}$, M Santos ${ }^{1,2}$, CZ Berton ${ }^{1}$, CL Chiarella ${ }^{1}$, MS Tanada ${ }^{1}$, EB Cordts ${ }^{1,2}$, WP de Carvalho ${ }^{1,2}$, CP Barbosa ${ }^{1,2}$

${ }^{1}$ Instituto Ideia Fertil de Saúde Reprodutiva, Santo André - SP, Brazil.

${ }^{2}$ Faculdade de Medicina do ABC, Santo André - SP, Brazil.

Objective: The transfer of euploid embryos allows better implantation rates. In Brazil, evaluation of morphology is performed to select the best embryo, since genetic analysis is still a high-cost implement. The study aimed to evaluate if there is a correlation between morphology of trophectoderm and its chromosomal status.

Methods: 113 blastocysts formed in D5/D6 from 58 cycles of in vitro fertilization from January 2016 to May 2017 were evaluated. All patients with indication for PGD/ PGS were included in this study. The mean age of women was $37.04( \pm 5.65)$. Biopsied blastocysts were classified according to their morphology. Cells were sent for genetic analysis using the CGH array, SNP array or NGS techniques. Statistical analysis was performed using the chi-square test, considering $P \leq 0.05$.

Results: 44 (38.9\%) blastocysts were euploid after chromosome analysis. The blastocysts with trophectoderm grade A presented $71.43 \%$ of euploidy, those with grade $B$, $60 \%$ and those with grade C, $19.67 \%(P \leq 0.05)$.

Conclusion: Although the best trophectoderm morphology scores show the highest euploidy rates, only this evaluation is not enough to guarantee the genetic viability of the embryo.

\section{0-05. Oral Dydrogesterone vs. vaginal progesterone capsules for luteal-phase support in women undergoing embryo transfer: a systematic review and meta-analysis}

MWP Barbosa ${ }^{1,2}$, NPB Valadares ${ }^{1}$, ACP $^{\text {Barbosa }}{ }^{1}$, AS Amaral $^{1}$, JR Iglesias ${ }^{1}$, CO Nastri ${ }^{3}$, WP Martins ${ }^{3}$

${ }^{1}$ Genesis - Centro de Assistência em Reprodução Humana - Brasília, DF.

${ }^{2}$ FMRP - USP - Faculdade de Medicina de Ribeirão Preto Ribeirão Preto, SP.

${ }^{3}$ SEMEAR fertilidade - Ribeirão Preto, SP.

Objective: To identify, appraise and summarize the evidence from randomized controlled trials (RCTs) comparing oral dydrogesterone with vaginal progesterone capsules for luteal-phase support (LPS) in women undergoing assisted reproduction techniques: fresh or frozen embryo transfer following in vitro fertilization.

Methods: Two independent authors performed the initial record screening based on title and abstracts, study selection, data extraction, and evaluation of the risk of bias. The comparison between dydrogesterone and progesterone were summarized as risk ratio (RR) and the precision of the estimates was assessed by the $95 \%$ confidence interval (CI).
Results: Our electronic search was performed in Jun-072017. Our electronic searches retrieved in 376 records and 9 studies were considered eligible and included in the systematic review and quantitative analysis. There is good quality evidence that oral dydrogesterone provided at least similar results than vaginal progesterone capsules on live birth/ongoing pregnancy $(\mathrm{RR}=1.08,95 \% \mathrm{CI}=0.92$ $1.26, \mathrm{I}^{2}=29 \%, 8$ RCTs, 3,386 women), and on clinical pregnancy (RR 1.10, 95\% CI 0.95 to 1.27 ; I2 $=43 \%$; 9 RCTs; 4,061 women). Additionally, we found moderate quality evidence of no relevant difference on miscarriage ( $R R=0.92,95 \% C I=0.68-1.26, \mathrm{I}^{2}=6 \%, 8 \mathrm{RCTs}, 988$ clinical pregnancies; the quality of the evidence was downgraded because of imprecision).

Conclusion: There is good quality evidence from RCTs suggesting that oral dydrogesterone provides at least similar reproductive outcomes than vaginal progesterone capsules when used for LPS in women undergoing embryo transfer. Such medication should be considered a good reasonable option and the choice between one or another should be based on costs and side-effects.

\section{0-06. The first case in South America of pre-implantation ge- netic diagnosis to select com- patible embryo for cord blood transplantation as treatment for sickle cell anemia}

\author{
CD Martinhago ${ }^{1}$, KRN Endo $^{1}$, MA Oliveira ${ }^{1}$, AMM Dias ${ }^{1}$, \\ GS Pereira ${ }^{1}$, AM Azzolini ${ }^{1}$, PRQ Estrada ${ }^{1}$, CG Bruzaca ${ }^{1}$, \\ ACN Martinhago ${ }^{1}$
}

${ }^{1}$ Chromosome Medicina Genômica Laboratory, São Paulo, $S P$, Brazil.

Introduction: Sickle cell anemia (MIM\#603903) is an inherited systemic hemoglobinopathy that affects the hemoglobin production in red blood cells leading to early morbidity and mortality. It is caused by a homozygous nucleotide substitution (c.20A>T) in the $\beta$-globin gene (HBB) that changes a glutamic acid to a valine in the protein. We present a case report of a fertile couple, both carriers of the sickle cell anemia mutation, with an affected daughter.

Case Report: Six cycles of assisted reproductive techniques were performed resulting in 53 embryos in cleavage stage. Each embryo were biopsied and analyzed for pre-implantation genetic diagnosis (PGD) by fluorescent polymerase chain reaction using polymorphic markers of the region of interest followed by capillary electrophoresis in an automated genetic analyzer. HLA Compatible and normal embryos for the mutation represented $3(5.66 \%)$; while the carriers and compatible $6(11.32 \%)$; therefore compatible embryos with daughter affected represented $9(16.98 \%)$. Embryo in blastocyst stage was transferred, resulting in a healthy male newborn who had the umbilical cord blood cells collected and stored. The affected daughter was immunosuppressed and received transplated cells from the umbilical cord blood of her brother; the treatment was successful.

Comments: Selecting embryos with PGD technologies indicate the most effective treatment plan for parents who want to have a healthy child and would cure another children already affected by inherited hemoglobinopathy. 


\section{0-07. Seminal profile of $\mathbf{2 3 . 5 0 4}$ patients over the years: 7 years of experience}

\author{
C Nogueira ${ }^{1}$, IH Yoshida ${ }^{1,2}$, FL Vilarino ${ }^{1}$, WP de Car- \\ valho $^{1,2}$, EB Cordts ${ }^{1,2}$, CP Barbosa ${ }^{1,2}$ \\ ${ }^{1}$ Instituto Ideia Fértil de Saúde Reprodutiva, Santo André \\ - SP, Brazil. \\ ${ }^{2}$ Faculdade de Medicina do ABC, Santo André - SP, Brazil.
}

Objetive: Evaluate the behavior of seminal parameters over the years 2010 to May 2017.

Methods: A retrospective study, realized from January 2010 to May 2017, covering men who underwent sperm examination. Seminal parameters (volume, sperm concentration, motility, morphology) of 23,504 men were evaluate, besides the age and the time of infertility (in years). The groups were compare to each other in order to observe if there were modifications of the seminal parameters in that period.

Results: There was no change over the years considering the time of infertility, being the average of 3.78 years. With respect to age, the mean of the patients is 36.53 years, with a trend of increase of 0.2 years. In relation to the volume of seminal samples, the mean is $3.29 \mathrm{~mL}$, with a trend of decrease in $0.05 \mathrm{~mL}$. Already sperm concentration, the average is $34.37 \mathrm{million} / \mathrm{mL}$, with a decrease trend of 1.0 million $/ \mathrm{mL}$. Progressive sperm motility showed an average of $47.27 \%$ and there is a decrease trend of $0.67 \%$. Finally, sperm morphology presented an average of $2.79 \%$ of normal spermatozoa, with a trend of decrease of $0.33 \%$. Conclusion: It can be noted that over the years, the sperm quality of men looking for assisted reproduction clinics has tended to decrease in macroscopic and microscopic parameters (volume, sperm concentration, motility and morphology).

\section{0-08. Vitrification of ovarian tissue and heterotopic autolo- gous transplantation in prepu- bertal Wistar rats}

\author{
L Wietowsky ${ }^{1}$, D Til ${ }^{1}$, RA Salvador ${ }^{1}$, NLL Amaral ${ }^{2}$, AP \\ Senn ${ }^{1}$, VLL Amaral ${ }^{1}$
}

${ }^{1}$ Laboratório de Biotecnologia da Reprodução (LBR), Universidade do Vale do Itajaí (UNIVALI), Itajaí, Santa Catarina, Brazil.

${ }^{2}$ Centro Clínico Veterinário (CCV), Itajaí, Santa Catarina, Brazil.

Objective: To evaluate the efficiency of heterotopic autografting of ovarian tissue after vitrification in prepubertal rats.

Methods: Fragments of excised ovaries of prepubertal rats were used after assessing cellular viability post warming in order to determine the best vitrification protocol prior to retro-auricular autografting. Prepubertal females $(\mathrm{N}=24)$ were castrated and divided into Group 1 - transplantation of the fresh ovarian tissue; Group 2 - transplantation of vitrified/warmed tissue; Group 3 - bilateral oophorectomy without transplantation. The ovarian fragments were exposed to solutions of the commercial kit Ingamed ${ }^{\circledR}$, allocated in bacteriological loops and immersed in liquid nitrogen. After 60 days of transplantation, a vaginal mucosa cytology was performed, followed by euthanasia, cardiac puncture of a blood sample for luteinizing hormone and estradiol levels determinations, and excision of the transplanted fragment for histology.

Results: Vaginal cytology revealed that $87.5 \%$ of females of Group 1 and 2 had estrus while all females in Group 3 remained in diestrus. The mean $\mathrm{LH}$ value of Group $1(0.08 \mathrm{mIU} / \mathrm{mL})$ and Group $2(0.34 \mathrm{mIU} / \mathrm{mL})$ were statistically different from that of Group 3 (2.27 $\mathrm{mIU} / \mathrm{mL}$ ). E2 values did not differ between groups. The histological analysis of excised grafts of Group 1 versus Group 2 showed a higher percentage of primary follicles ( $62.5 \%$ vs. $12.5 \%)$, developing follicles (75\% vs. $25 \%)$, corpus luteum (37.5\% vs. $12.5 \%)$ and stromal region ( $100 \%$ vs. $87.5 \%)$.

Conclusion: This study indicate that vitrification of pre-pubertal ovarian tissue can be used to preserve fertility and to restore endocrine function in castrated rats.

\section{0-09. Wistar rats immature testicular tissue vitrification and heterotopic grafting} Tames $^{1}$, NLL Amaral ${ }^{2}$, VLL Amaral ${ }^{1}$

${ }^{1}$ UNVALI - Universidade do Vale do Itajai, Itajaí- SC, Brazil.

${ }^{2} \mathrm{CCV}$ - Centro Clínico Veterinário, Itajaí - SC, Brazil.

Objective: To evaluate the efficiency of two vitrification protocols for rat immature testicular tissue and heterotopic transplantation.

Methods: Twenty-four pre-pubertal Wistar rats were divided into three groups $(n=8)$. After orchiectomy, testicular fragments $(3 \mathrm{~mm})$ from Group 1 and 2 were vitrified with different cryoprotectants concentration solutions, using sterile inoculation loops as support. After warming fragments were submitted to cell viability assessment by Trypan blue and histological evaluation. Vitrified (Groups 1 and 2) and fresh (Group 3) fragments were grafted to the animals periauricular region. After 8 weeks of grafting, histological analysis of implant site was performed.

Results: Viability recovery rate from Group 1 (72.09\%) was higher $(p=0.02)$ than from Group $2(59.19 \%)$. Histological analysis showed similar tubular integrity between fresh fragments of Group 1 and 3. Group 2 samples presented lower tubular integrity. Histological analysis from grafts of groups was performed. In all groups it was possible to observe the implant site, however, no fragment of testicular tissue or signs of inflammation were histologically observed in most samples of Group 1 and 3. In one sample of Group 2 was observed the presence of degenerated seminiferous tubules with necrosis and signs of inflammatory process. In another sample of Group 2, seminiferous tubules could be observed in the implant site.

Conclusion: The vitrification of pre-pubertal testicular tissue of rats showed little damage in cell viability and histological analysis when cryoprotectants were used in a lower concentration. Heterotopic transplantation could not preserve the structural organization of the testicular tissue. 